\title{
Fourier transform infrared
} spectrophotometer with attenuated total reflectance (FTIR-ATR) for analysis of uroliths in caprine species

\author{
Anvitha Hansoge1, Sudheesh S Nair ${ }^{2}$, M.V. Chinnu ${ }^{3}$, \\ M.K. Narayanan ${ }^{4}$ and K.D. John Martin ${ }^{5}$ \\ Department of Veterinary Surgery and Radiology, College of Veterinary and Animal Sciences, \\ Mannuthy, Thrissur, Kerala Veterinary and Animal Sciences University, India
} Citation: Anvitha, H., Nair, S.S., Chinnu,M.V., Narayanan, M.K. and John Martin, K.D. 2021. Fourier Transform Infrared Spectrophotometer with attenuated total reflectance (FTIR-ATR) for analysis of Uroliths in caprine species. J. Vet. Anim. Sci. 52(2): 125-130.

DOI: https://doi.org/10.51966/jvas.2021.52.2.125-130

Received: 8.12.2020

Accepted: 10.01.2021

Published: 01.06.2021

\begin{abstract}
Pathophysiology of obstructive urolithiasis in goats are multifactorial involving management, nutritional and hormonal factors. Analysis of the composition of a particular urolith would aid in understanding the aetiopathogenesis of the calculi which can facilitate establishment of appropriate treatment and prevention protocols. Four cases of chronic obstructive urolithiasis in male goats presented to University Veterinary Hospital were surgically managed by tube cystotomy. The calculi extracted from the bladder were subjected to Fourier transform infrared spectrophotometer with attenuated total reflectance (FTIR-ATR) to identify the composition of the calculi. The infrared wavelength bandwidths of $\mathrm{H}-\mathrm{O}-\mathrm{H}$ stretching vibrations, $\mathrm{H}-\mathrm{O}-\mathrm{H}$ bending modes of vibrations of water molecules, $\mathrm{N}-\mathrm{H}$ symmetric stretching vibrations, $\mathrm{N}-\mathrm{H}$ bending vibration and $\mathrm{N}-\mathrm{H}$ asymmetric bending vibration in $\mathrm{NH} 4+$ unit's ionic phosphate were compared with the available reference spectrum of wavelength and were found to be identical and comparable with the standard infrared wavelength of struvite calculi.
\end{abstract}

Key words: Fourier transform infrared spectrophotometer, Attenuated total reflectance, Chronic obstructive urolithiasis, Goats

Urolithiasis is a clinical condition resulting from multiple congenital and/or acquired pathophysiologic processes that result in an elevated concentration of insoluble crystalloids in the urine (Dar, 2011). Pathophysiology of obstructive urolithiasis were identified to be multifactorial

Part of M.V.Sc. thesis submitted by the first author to the Kerala Veterinary and Animal Sciences University, Pookode, Wayanad, Kerala

1. M.V.Sc. student email : anvi.hansoge@gmail.com Ph. 9590125001

2. Assistant Professor

3. Assistant Professor, Department of Veterinary Biochemistry

4. Professor

5. Professor and Head

Copyright: (C) 2021 Anvitha Hansoge et al. This is an open access article distributed under the terms of the Creative Commons Attribution 4.0 International License (http://creativecommons.org/licenses/by/4.0/) which permits unrestricted use, distribution, and reproduction in any medium, provided the original author and source are credited. 
involving management, nutritional and hormonal factors. Sun et al. (2010) reported the most common aetiology of formation of urolithiasis as the supersaturation of the excretory solutes in the urine, increased concentrate feeding and lack of sufficient water intake and that the analysis of the composition of a particular urolith would aid in understanding the aetiopathogenesis of the calculi and further facilitated establishment of appropriate treatment and prevention protocols. Primiano et al. (2014) reported that FT-IR was found to be the gold standard test for analysis of urinary calculi and that it showed a high sensitivity thereby giving an accurate recognition of the composition of each stone.

\section{Materials and Methods}

Four male goats with chronic obstructive urolithiasis with an intact bladder were presented to University Veterinary Hospital, Mannuthy with history of stranguria. All animals had the history of being fed with high concentrate diet (cattle ration). The Ultrasonography evaluation revealed hypoechoic turgid bladder with hyper echoic uroliths forming sludge in the bladder floor that made wavy motion on gentle succession. (Fig-1) The animals underwent tube cystostomy by standard procedure documented by Gazi et al. (2014). The urinary calculi was collected by sieving the urine (Fig-2) The microanalysis of the sediments revealed uroliths similar to struvite crystals. (Fig- 3)

The urolith samples were serially numbered as $A_{1}, A_{2}, A 3$ and $A 4$ respectively. The calculi were thoroughly washed with deionized water to remove the adhered dirt and blood clots, dried at $37^{\circ} \mathrm{C}$ and stored for analysis of the mineral content. Analysis of calculi was done using Perkin Elmer Spectrum 2 Infra-red Spectrophotometer - USA using the principle of Fourier transform infrared spectrophotometer with Attenuated Total Reflectance (FTIR-ATR) at Central Instrumentation Laboratory, College of Veterinary and Animal Sciences, Mannuthy. To create a background blank in the machine, $300 \mathrm{mg}$ of potassium bromide $(\mathrm{KBr})$ was pressed under pressure to form a pellet and analysed. (Fig-3) Subsequently, $298 \mathrm{mg}$ of $\mathrm{KBr}$ and $2 \mathrm{mg}$ of powdered uroliths were mixed and was pressed under pressure and the pellet was scanned and analysed to record the spectrum using FTIR- ATR machine and the observed spectrum was compared to the standard reference spectrum based on the observed transmittance and frequency of the waveforms.

\section{Results and Discussion}

The results of analysis of calculi in Fourier Transform Infrared Spectrometry with Attenuated Total Reflectance (FTIR-ATR) are depicted in Table 1. Analysis of the calculi from animal no. $A_{1}, A_{2}, A 3$ and A4 using FTIR-ATR revealed a graph with an IR spectrum ranging from 4000 to $450 \mathrm{~cm}^{-1}$. The $\mathrm{H}-\mathrm{O}-\mathrm{H}$ stretching vibrations of water of crystallization were at $3401.94 \mathrm{~cm}^{-1}$ for animal no $A_{1}, 3391.81 \mathrm{~cm}^{-1}$ for animal no $A_{2}, 3500$ to $3350 \mathrm{~cm}^{-1}$ for animal no $A_{3}$ and $3360.29 \mathrm{~cm}^{-1}$ for animal no $A_{4}$. The $\mathrm{H}-\mathrm{O}-\mathrm{H}$ stretching vibrations of the cluster of water molecules were at 2231.52, 2346.67 and $2321.69 \mathrm{~cm}^{-1}$ for animals' $A_{1} A_{2} \quad A_{3} A_{4}$ respectively. The $\mathrm{H}-\mathrm{O}-\mathrm{H}$ bending modes of vibrations ranged from 1440.19, 1434.90, 1434.68 and $1441.17 \mathrm{~cm}^{-1}$ for animals' $A_{1} A_{2} A_{3}$ $A_{4}$ respectively. The $\mathrm{N}-\mathrm{H}$ symmetric stretching vibrations were at $3401.94 \mathrm{~cm}^{-1}$ for animal no $A_{1}, 3391.81 \mathrm{~cm}^{-1}$ for animal no $A_{2}, 3500$ to $3350 \mathrm{~cm}^{-1}$ for animal no $A_{3}$ and $3360.29 \mathrm{~cm}^{-1}$ for animal no $A_{4}$. The $\mathrm{N}-\mathrm{H}$ bending vibration was at 1670.28, 1650.70, 1651.8 and 1654.67 $\mathrm{cm}^{-1}$ for animals' $A_{1} A_{2} A_{3} A_{4}$ respectively and the $\mathrm{N}-\mathrm{H}$ asymmetric bending vibration in $\mathrm{NH} 4+$ unit's ionic phosphate was at 995.16, 1000.4, 1000.48 and $1000.14 \mathrm{~cm}^{-1}$ for animals A1 A2 A3 A4 respectively. These infrared wavelength bandwidths were compared with the available reference spectrum of standard wavelength of struvite calculi proposed by Bindhu and Thambi (2012). The infrared spectrum from animals are depicted in the figure 5-8.

The spectra contained a bandwidth comparable to that of the standard IR spectra of struvite crystals as proposed by Bindhu and Thambi (2012) who reported that in a pure magnesium ammonium phosphate spectrum, the broad envelop at $3270 \mathrm{~cm}^{-1}$ correlates to the $\mathrm{O}-\mathrm{H}$ and $\mathrm{N}-\mathrm{H}$ stretching vibrations. The band at $2935 \mathrm{~cm}^{-1}$ is due to $\mathrm{NH} 4+$ ion and the 


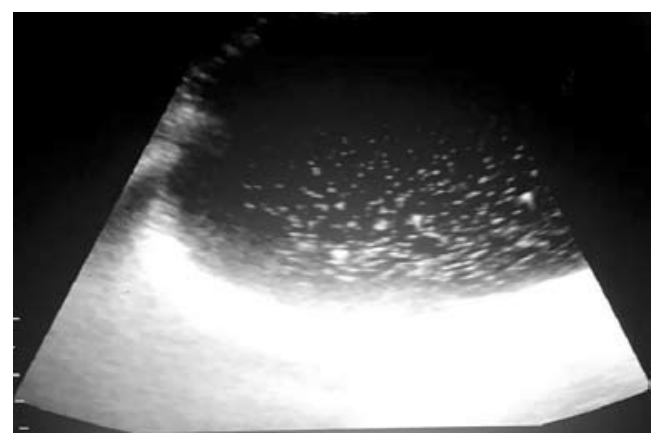

Fig-1 Ultrasonography to detect uroliths

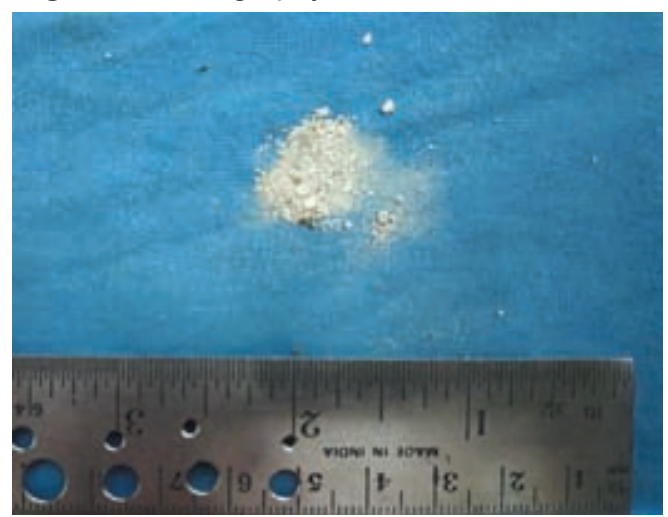

Fig-2 Uroliths retrieved from urinary bladder by tube cystostomy

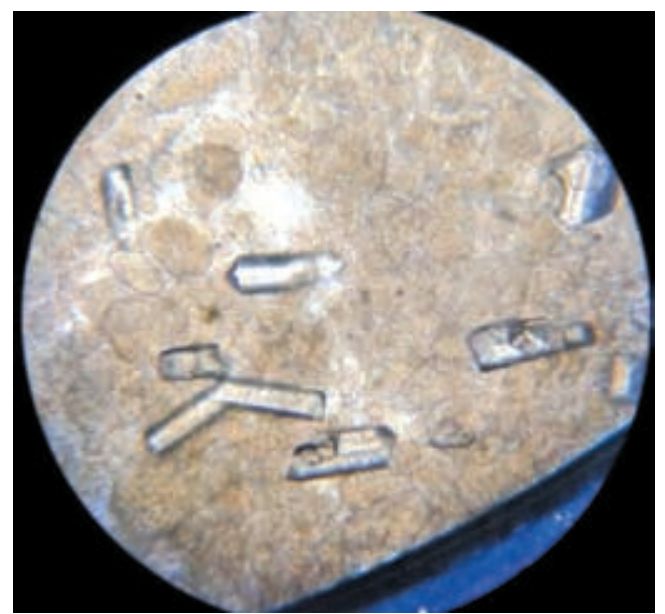

Fig-3 Microscopic examination of struvute cystals

band corresponding to $1666 \mathrm{~cm}^{-1}$ was assigned to $\mathrm{N}-\mathrm{H}$ bending vibrations. The sharp yet weak envelop correlating to $1445 \mathrm{~cm}^{-1}$ could be due to N-O asymmetric stretching vibration. The absorption band occurring at $1010 \mathrm{~cm}^{-1}$ was assigned to ionic phosphate.

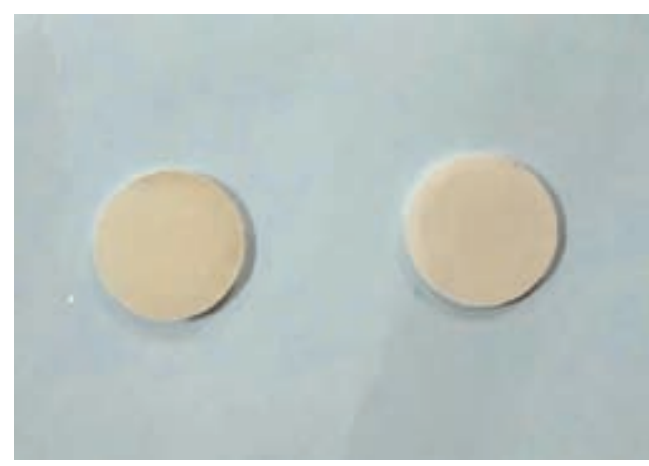

Fig-3 Prepared pellets of $\mathrm{KBr}$ and urolith for FTIR - ATR spectroscopy

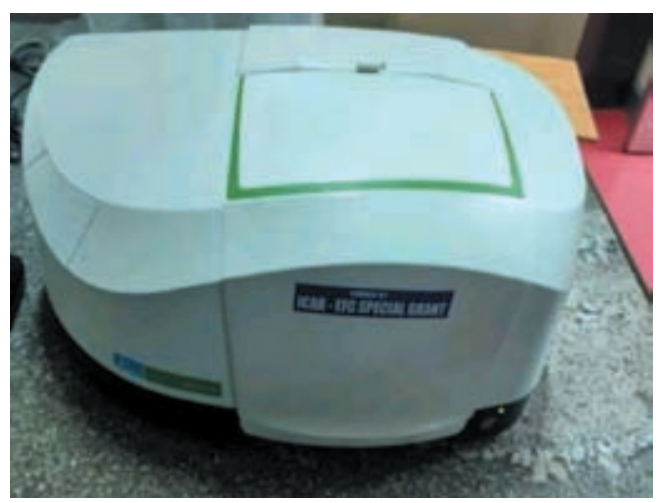

Fig-4 Perkin Elmer Spectrum 2 Infra-red Spectrophotometer- USA

The determination of molecular composition, crystalline composition and the quantification of all stone components are helpful to establish the etiology of stones disease and consequently aid in the treatment of the clinical disease. Various methodologies exist for stone analysis. Fourier transform infrared spectroscopy (FT-IR) is becoming the gold standard for stone analysis as it is the most appropriate technique. The principle of FT-IR involves generating an infrared spectrum from the vibrational motion of the molecules. These vibrational frequencies are unique for each compound. This feature of the spectrometer is used to characterize inorganic compounds from organic ones present in the calculi. The band intensities are proportional to the compound concentration and hence qualitative estimations are also obtained. FT-IR spectroscopy leads to unambiguous information about the stone composition, both for main substances and trace elements, all essentials to guide therapy. 


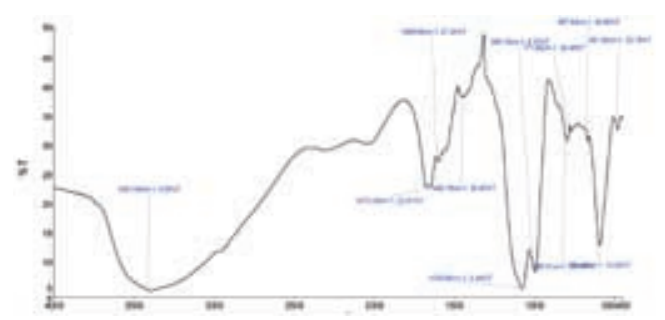

Figure 5: Infrared spectrum from goat $A_{1}$ showing struvite bands at 3401, 1440, 1670 and $995.16 \mathrm{~cm}^{-1}$

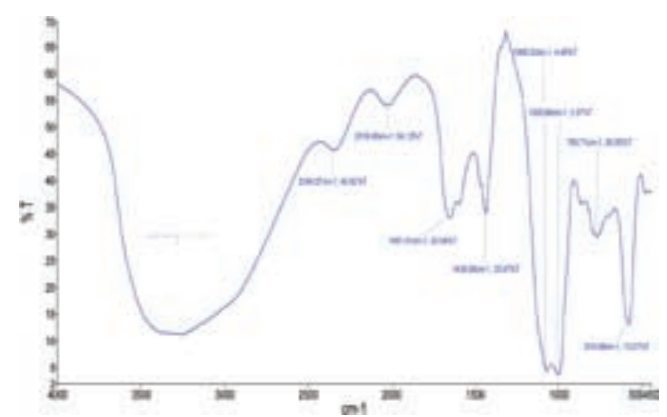

Figure 7: Infrared spectrum from goat $A_{3}$ showing struvite bands at 3500-3350, 1434, 2346, 1 and $1000.48 \mathrm{~cm}^{-1}$

Wang et al. (2009) explained the preparation of the calculi before spectroscopy by washing the crystals twice in deionising water and air drying overnight. The authors recorded strong bands of wavelength 556.1, 986.4, 1066.7, $3201.7 \mathrm{~cm}^{-1}$ and medium-strong bands at $1673.9,2385.2 \mathrm{~cm}^{-1}$ which was conclusive of magnesium ammonium phosphate crystals. Bindhu and Thambi (2012), in their study on microanalysis of the struvite crystal, reported that the standard IR spectra ranged within 4000 to $450 \mathrm{~cm}^{-1}$ for pure struvite with the broad envelop at 3270 correlated to the $\mathrm{O}-\mathrm{H}$ and $\mathrm{N}-\mathrm{H}$ stretching vibrations. In their study, the band at $2935 \mathrm{~cm}-1$ was due to $\mathrm{NH} 4+$ ion and the band corresponding to $1666 \mathrm{~cm}-1$ was assigned to $\mathrm{N}-\mathrm{H}$ bending vibrations. The sharp yet weak envelop of $1445 \mathrm{~cm}-1$ was due to $\mathrm{N}-\mathrm{O}$ asymmetric stretching vibration. The absorption band occurred at $1010 \mathrm{~cm}-1$ was assigned to ionic phosphate.

In the present study based on the alkaline $\mathrm{pH}$ of urine in all animals and the confirmed composition of struvite, medical

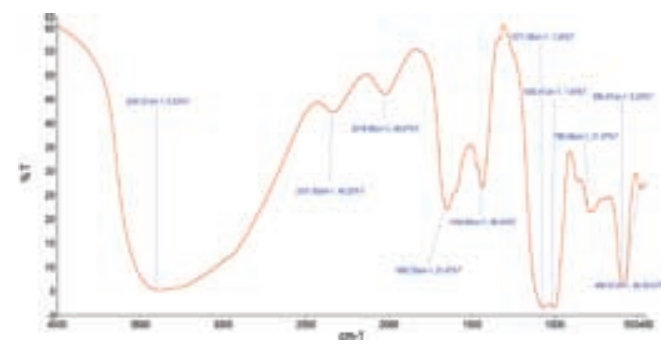

Figure 6: Infrared spectrum from goat $A_{2}$ showing struvite bands at 3391, 1434, 2331, 1650 and $1000.4 \mathrm{~cm}^{-1}$

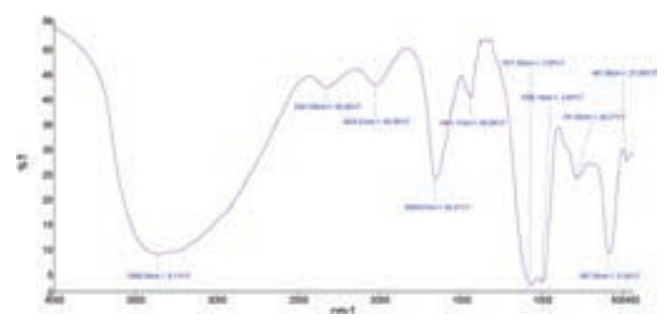

Figure 8: Infrared spectrum of goat $A_{4}$ showing struvite bands at 3360, 1441, 2321, 1654 and $1000.14 \mathrm{~cm}^{-1}$

management was done with ammonium chloride at $300 \mathrm{mg} / \mathrm{kg}$ body weight per orally and the owners were advised to avoid feeding cattle feed to goats and provide ad libitum water.

\section{Conclusion}

Urolithiasis is a clinical disease affecting the feedlot animals fed on high concentrate diet (cattle ration). The most common urolith manifested in male goats is magnesium ammonium phosphate (struvite) calculi. The use of Fourier transform infrared spectroscopy has been highly specific for determining the composition of the urolith which invariably has aided in specific treatment protocols and preventive management strategies in caprine chronic obstructive urolithiasis.

\section{Acknowledgement}

Central Instrument Laboratory, CVAS, Mannuthy. 
Table 1. Results of analysis of calculi in Fourier Transform Infrared Spectrometry with Attenuated Total Reflectance (FTIR-ATR)

\begin{tabular}{|c|c|c|c|}
\hline Functional Group Assignments & $\begin{array}{l}\text { Animal } \\
\text { no. }\end{array}$ & $\begin{array}{l}\text { Reported IR } \\
\text { wavelength } \\
\quad\left(\mathrm{cm}^{-1}\right)\end{array}$ & $\begin{array}{l}\text { Standard IR wavelength of } \\
\text { pure struvite }\left(\mathrm{cm}^{-1}\right)(\text { Bindhu } \\
\text { and Thambi, 2012) }\end{array}$ \\
\hline \multirow{4}{*}{$\begin{array}{l}\mathrm{H}-\mathrm{O}-\mathrm{H} \text { stretching vibrations of water } \\
\text { of crystallization }\end{array}$} & $A_{1}$ & 3401.94 & \multirow{4}{*}{3270} \\
\hline & $A_{2}$ & 3391.81 & \\
\hline & $\mathrm{A}_{3}$ & $3500-3350$ & \\
\hline & $\mathrm{A}_{4}$ & 3360.29 & \\
\hline \multirow{4}{*}{$\begin{array}{l}\mathrm{H}-\mathrm{O}-\mathrm{H} \text { stretching vibrations of a } \\
\text { cluster of water molecules }\end{array}$} & $A_{1}$ & - & \multirow{4}{*}{2385} \\
\hline & $A_{2}$ & 2331.52 & \\
\hline & $\mathrm{A}_{3}$ & 2346.67 & \\
\hline & $\mathrm{A}_{4}$ & 2321.69 & \\
\hline \multirow{4}{*}{$\mathrm{H}-\mathrm{O}-\mathrm{H}$ bending modes of vibrations } & $A_{1}$ & 1440.19 & \multirow{4}{*}{1445} \\
\hline & $A_{2}$ & 1434.90 & \\
\hline & $\mathrm{A}_{3}$ & 1434.68 & \\
\hline & $\mathrm{A}_{4}$ & 1441.17 & \\
\hline $\begin{array}{l}\mathrm{N}-\mathrm{H} \text { symmetric stretching vibrations } \\
\text { in } \mathrm{NH} 4+\text { units }\end{array}$ & & - & 2935 \\
\hline \multirow{4}{*}{$\mathrm{N}-\mathrm{H}$ symmetric stretching vibrations } & $A_{1}$ & 3401.94 & \multirow{4}{*}{3270} \\
\hline & $\mathrm{A}_{2}$ & 3391.81 & \\
\hline & $\mathrm{A}_{3}$ & $3500-3350$ & \\
\hline & $\mathrm{A}_{4}$ & 3360.29 & \\
\hline $\begin{array}{l}\mathrm{N}-\mathrm{H} \text { symmetric stretching vibrations } \\
\text { in } \mathrm{NH} 4+\text { units }\end{array}$ & & - & 2935 \\
\hline \multirow{4}{*}{$\mathrm{N}-\mathrm{H}$ bending vibration } & $A_{1}$ & 1670.28 & \multirow{4}{*}{1666} \\
\hline & $\mathrm{A}_{2}$ & 1650.70 & \\
\hline & $\mathrm{A}_{3}$ & 1651.8 & \\
\hline & $\mathrm{A}_{4}$ & 1654.67 & \\
\hline \multirow{4}{*}{$\begin{array}{l}\mathrm{N}-\mathrm{H} \text { asymmetric bending vibration in } \\
\mathrm{NH} 4+\text { units } \\
\text { lonic phosphate }\end{array}$} & $A_{1}$ & 995.16 & \multirow{4}{*}{1010} \\
\hline & $A_{2}$ & 1000.4 & \\
\hline & $\mathrm{A}_{3}$ & 1000.48 & \\
\hline & $\mathrm{A}_{4}$ & 1000.14 & \\
\hline
\end{tabular}




\section{References}

Bindhu, B. and Thambi, T.A. 2012. Formation and microanalysis of struvite urinary calculi. Int. J. Eng. Res. Appl. 2: 14801485.

Dar, F.A. 2011. Evaluation and management of urolithiasis in goats. MVSc thesis, Kerala Veterinary and Animal sciences University, Pookode. 1- 34p.

Gazi, M.A., Makhdoomi, D.M., Parrah, J.D., Ganai, A.M., Shiekh, G.N. and Mir, S.A. 2014. Recent advances in surgical management of urolithiasis in sheep and goat. Afr. J. Agri. Res. 9: 2055-2061.

Primiano, A., Persichilli, S., Gambaro, G., Ferraro, P.M., D’Addessi, A., Cocci,
A., Schiattarella, A., Zuppi, C. and Gervasoni, J. 2014. FT-IR analysis of urinary stones: a helpful tool for clinician comparison with the chemical spot test. Disease Markers, 2014.

Sun, W., Wang, J., Zhang, K. and Wang, X. 2010. Study on precipitation of struvite and struvite- $K$ crystal in goats during onset of urolithiasis. Res. Vet. Sci. 88: 461-466.

Wang, J., Sun, W. and Wang, X. 2009. Comparison of effect of high intake of magnesium with high intake of phosphorus and potassium on urolithiasis in goats fed with cottonseed meal diet. Res. Vet. Sci. 87: 79-84. 